\title{
Geometric and Physical Modeling of Natural Intelligence
}

\author{
Reji M. Issac
}

\begin{abstract}
Intelligence is defined as what we do when we do not know what to do. When we look in to the in depth structure of knowledge, its generation and about its existence, we can understand the existence of seven pillars of knowledge through which spreading of the hexagonal words generates pure knowledge which exists in an eternal world. This paper is bringing a geometric and physical model for natural intelligence using $\mathrm{CW}$ (Computing with Words) of Cybernetics which can be adapted to machines and robotics giving them the capability to process natural intelligence over artificial intelligence in an age where machines are connected to Noosphere. When the heart produces each word even the numbers of alphabets are fixed in this order.
\end{abstract}

Keywords--- Modeling, CW, Cybernetics, Shape Modeling, Parametric Modeling, Geometry

\section{INTRODUCTION}

$\mathrm{I}$ NTELLIGENCE defined in many ways based on capacity for logic, abstraction, understanding, communication, learning, emotional knowledge, memory, creativity and problem-solving. It can also be more generally described as the ability to perceive and/or retain knowledge or information and apply it to itself or other instances of knowledge or information. In most simplified terms intelligence can be defined as what we do when we do not know what to do. Intelligence is most widely studied in humans, but has also been observed in non-human animals and in plants Sternberg and Salter (1982). Artificial Intelligence is the simulation of intelligence in machines. The etymology of the word intelligence derived from the Latin verb 'intelligere' Johnston (2008). 'Intellectus' is the term for understanding which is translation for the Greek term 'nous'. This term was however strongly linked to the metaphysical and cosmological theories of teleological scholasticism, including theories of the immortality of the soul, and the concept of the Active Intellect (also known as the Active Intelligence) Chardin (1948) ; Johnston (2008).

Human cognition is central to decision making. Here brain is the central part which receives information from various sources through the various senses. If we have a fundamental understanding of the process of cognition and inherent weaknesses in the process, we will be better able to compensate for weaknesses in this process and arrive at better decisions in the long run. Cybernetics is known as the art and science of understanding, which is the science related with the intellect, where the basic principle is regulations through

Reji M. Issac, B.P.C. College, Baselios Mount, Piravom(P.O.), Ernakulam, Kerala, India.E-mail:rejimissac@ieee.org DOI: 10.9756/BIJMMI.8137 control and feedback using communications in living beings and machines Wiener (1948). It is also an interdisciplinary and multidisciplinary subject that is working as a coordinating subject for which it is also known as the science of government. This paper is giving an idea about a branch of science known as Noology, where the word is derived from the Greek words voṽ "logos". Noology or cognitive neuroscience is the systematic study and organization of everything dealing with knowing and knowledge. It is also used to describe the science of intellect and intellectual phenomena. It is the study of images of thought, their emergence, their genealogy, and their creation Issac(2010). The word Logos in Greek gave birth to the word Logic, the science of reasoning, which is more concerned with the processes and products of reasoning. Moreover, the course of its development from Aristotle to the present day has been more idiosyncratic and specialized than what the concept of a science of reason would suggest. As described in Issac(2010), Word, in the conventional sense, is a unit of language which functions as a principal carrier of meaning, consisting of one or more spoken sounds or their written representation. They are either the smallest units susceptible of independent use or consist of two or three smallest units combined under certain linking conditions which are composed of one or more morphemes. They are distinguished phonologically in many languages and usually separated by spaces in writing. The native speakers of a language usually regard word as the smallest isolable meaningful element of the language, although some linguists would analyze these further into morphemes, which is an instance of vocal intercourse through the units of speech or writing. Here well-ordered words have the structure of a honeycomb with hexagonal shape as that of a pure hexagonal water molecule as in a snow flake.

Computing with Words is defined, to be a specific generalization of fuzzy logic, which admits self reference Rubin (1999). Randomization of declarative knowledge, which yields procedural knowledge, is $\mathrm{CW}$ (Computing with words).Generally computing is centered on manipulation of numbers and symbols. Computing with words or $\mathrm{CW}$, is a methodology in which the objects of computation are words and propositions drawn from a natural language, e.g., small, large, far, heavy, not very likely, the price of gas is low and declining, Piravom is near Kochi, it is very unlikely that there will be a significant increase in the price of oil in the near future, etc. Computing with words is inspired by the remarkable human capability to perform a wide variety of physical and mental tasks without any measurements and any computations Zadeh (2002).Parking a car, driving in heavy traffic, playing golf, riding a bicycle, understanding speech and summarizing a story are examples of such tasks. The 
brain's ability to manipulate perceptions like perceptions of distance, size, weight, color, speed, time, direction, force, number, truth, etc. underlies on this remarkable capability. The human recognition, decision and execution processes are based on Manipulation of perceptions. Computing with words (CW) provides a foundation for a computational theory of perceptions and perception-based rational decisions in an environment of imprecision, uncertainty and partial truth, as a methodology Zadeh (2002).

This paper is discussing about the physical and geometric structure of knowledge based on the seven pillars of knowledge Issac $(2012,2013)$ concept and how that is related with the natural intelligence and how we can model it to process with Artificial Intelligence to create more eco-friendly and user friendly machines world which can digest natural intelligence using $\mathrm{CW}$ (Computing with Words) of Cybernetics.

\section{COMPUTING WITH WORDS (CW)}

The usual method of computing is centered on manipulation of numbers and symbols. In contrast, computing with words, or CW for short, is a paradigm shift from the conventional computing, where in this methodology, the objects of computation are words and propositions drawn from a natural language. Out of the many misconceptions about what Computing with Words (CW) is and what it has to offer is the misconception about how closely $\mathrm{CW}$ is related to natural language processing Zadeh(2002).In reality, this is not the case. More importantly, at this juncture what is widely unrecognized is that moving from computation with numbers to computation with words has the potential for evolving into a basic paradigm shift - a paradigm shift which would open the door to a wide-ranging enlargement of the role of natural languages in scientific theories Zadeh (2002).

In essence, $\mathrm{CW}$ is a system of computation which adds two important capabilities to traditional systems of computation: (a) the capability to precisiate the meaning of words and propositions drawn from natural language; and (b) the capability to reason and compute with precisiated words and propositions Zadeh (2002). There are three principal components for a $\mathrm{CW}$ model. (a) A question, $\mathrm{Q}$, of the form: What is the value of a variable, Y? (b)An information set, I = $(\mathrm{p} 1, \ldots, \mathrm{pn})$, where the $\mathrm{pi}, \mathrm{i}=(1, \ldots, \mathrm{n})$, which are propositions individually or collectively are carriers of information about the value of $\mathrm{Y}$, that is, are question relevant. One or more of the pi may be drawn from world knowledge. A proposition, pi, assigns a value, vi, to a variable, $\mathrm{Xi}$, in pi.Pi is an answer to the question: What is the value of $\mathrm{Xi}$ ? $\mathrm{Xi}$ and vi ,which is explicit or implicit. A proposition, pi, may be unconditional or conditional, expressed as if - then rule. An assignment statement constrains the values which $\mathrm{Xi}$ is allowed to take. $\mathrm{Xi}$ and vi are referred to as the constrained variable and the constraining relation. More concretely, what this implies is that the meaning of a proposition, $\mathrm{p}$, may be represented as a generalized constraint, $\mathrm{X}$ isr $\mathrm{R}$, in which $\mathrm{X}$ is the constrained variable, $R$ is the constraining relation and $r$ defines the modality of the constraint, that is, the way in which $\mathrm{R}$ constrains $\mathrm{X}$. When vi is a word or combination of words, with vi being its linguistic value, $\mathrm{Xi}$ is referred to as a linguistic variable. When it is helpful to stress that pi assigns a value to a variable, pi is referred to as a valuation. Correspondently, the information set, I, is referred to as a valuation system, V. (c) The third component is an aggregation function, $f$, which relates $Y$ to the $X i$. ie. $Y=f$ (X1,.., Xn ).

The principal difference between $\mathrm{CW}$ and conventional systems of computation is that $\mathrm{CW}$ allows inclusion in the information set, I, of propositions expressed in a natural language, that is, linguistic variations. Legalization of linguistic variations has important implications. The information which in one or more respects is imprecise, uncertain, incomplete, unreliable, vague or partial are enhanced with the capability of computational methodologies bringing a method towards perfection. In realistic settings, such information is the norm rather than exception. Second, in cases in which there is a tolerance for imprecision, linguistic valuations serves to exploit the tolerance for imprecision, through the use of words in place of numbers Zadeh (2002). High level of machine intelligence, that is, high level of MIQ (Machine IQ) is facilitated through linguistic valuations which are close to human reasoning.

In this paper we attempt to map the three components of CW in to a triangular form Issac (2013)and this triangular description is extended over a total of six triangles which is part of a hexagon word consisting of six sides along with seventh centroid and hence deductions are made with forming other hexagons which can be considered as the fundamental geometric and physical structure of a word which exists in an eternal world. As described in the Bible, like a honey comb, well ordered words are forming knowledge. This hexagon structure is the fundamental structure of pure water available in nature, hexagonal water, and like the structure of hexagonal snowflakes, which are formed like from a mould. Structurally and geometrically this hexagon formed out of six subtriangles with unifying centroid can be considered as the basic structure of a word. The three sides of each triangle can be considered as the propositions and the derivations drawn from the propositions through the process of $\mathrm{CW}$.

\section{SEVEN PILLARS OF KNOWLEDGE}

A simple device for storage and retrieval of information which is often used in schools is the human brain which is the most complex system on earth. The human brain is changing functionally and structurally as a result of learning and experience. New neural connections increases possibility of learning, and remember and problem-solve capability which continue to form throughout life, particularly when human beings are in environments that are positive and stimulating and that encourage action and interaction Forester and Portage (2000) Issac (2010).

People develop cognitive strategies and effort-based beliefs about their intelligence when they are continually pressed to raise questions and to accept challenges, to find solutions that are not immediately apparent, to explain concepts, justify their reasoning, and seek information Prakash (1989). Education, which involves the process of developing 
right spirit, is used to liberate and develop more fully the habits of mind and the skills associated with them. The processes and concepts involved in the thought process in an integrated environment of body and spirit are presented here. Here thought is expressed through an active integrated environment of body and spirit instead of a passive "reflection" of an external "reality". The spreading of the words creates spirit, which forms the structure of knowledge in the hexagonal form.

Seven pillars of knowledge is a concept originally conceived from the Bible in proverbs 9:1. Here words are the building blocks of knowledge. After several years of research, experiences and revelations it was revealed that the seven pillars of knowledge, which itself we may be used to call as wisdom which are the well ordered words like honeycomb Issac (2010), are Truth, Justice, Love, Loyalty, Obedience, Intelligence and an invisible Wisdom which connects and unifies all together. If we place each of these pillars on the sides of a hexagon starting from top clockwise, we can place the unifying pillar of knowledge, Wisdom, in the centroid of the hexagon, which is shown as in Figure.1.

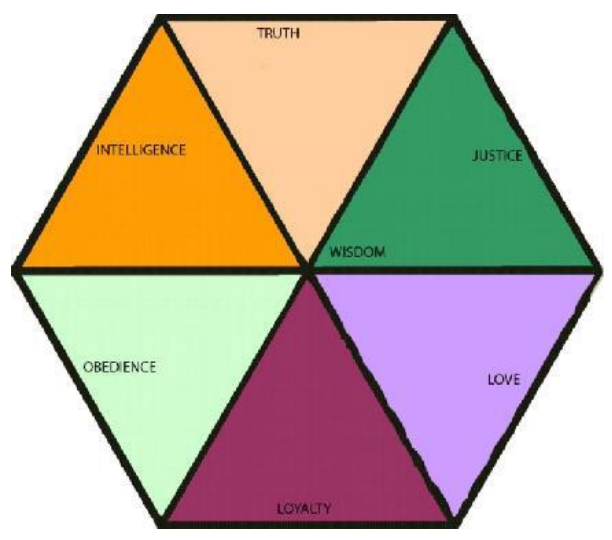

Figure 1: Geometric Structure of a Word

So we can deduce the physical and geometric structure of knowledge as in Figure.2. The science of Noology was developed to introduce the intellectual phenomena. Ideally Noology is considering all the possible forms and laws of intelligence. Modeling human and other minds and with fashioning a valid, fundamental, and universal theory of mind and cognitive phenomena is the concern of Noology. As a science of all actual and possible psyches and psychological phenomena, and the laws and behavioral manifestations, it is to be distinguished from psychology. The related field of artificial intelligence is the branch of computer science that endeavors to invest machines with mind and reason, or, ideally, that would create all possible types and degrees of intelligence Johnston (2008). One of the natural sub fields of Noology should be modeling ideation, and of artificial intelligence the automation of ideation, but for some mysterious reason mere traces of these sub fields are all that can so far be found in those disciplines.

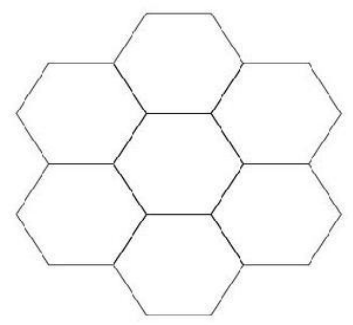

Figure 2: Geometric Structure of Knowledge

This paper is giving an idea about a branch of science known as Noology, where the word is derived from the Greek

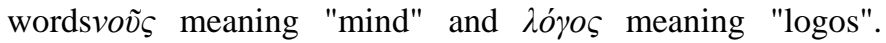
Noology outlines cognitive neuroscience. It is also used to describe the science of intellect and intellectual phenomena. It is the study of images of thought, their emergence, their genealogy, and their creation Issac (2010). Logic, ideally the science of reasoning, which is derived from the word Logos in Greek, is more concerned with the processes and products of reasoning. Moreover, the course of its development from Aristotle to the present day has been more idiosyncratic and specialized than what the concept of a science of reason would suggest.

\section{METHODOLOGY}

When we have the structure of the word and knowledge, we can develop a methodology to store and process the knowledge, which involves intelligence, where our target is to process natural intelligence compatible to the world of human and natural intelligence. Here we can connect the process using CW (Computing with Words) by subdividing each hexagonal word in to six triangles which represent Truth, Justice, Love, Loyalty, Obedience and Intelligence. The entire process is done and delivered through the unifying Wisdom, where wisdom is as defined in Proverbs 9:10 in the Bible. This structure also justifies the seven point evaluation process in the education sector. It is also found that the number of alphabets in a word of the English language fixed based on this standard through which an earth is constructed.

This way of computing with words makes machine intelligence compatible with the process that takes place in the heart of the people, through which the challenges and risks that we face in Artificial Intelligence can be avoided. Within this basic natural frame work we can also find optimum solutions for any computing needs. This way of computing also makes $\mathrm{CW}$ to closely work with natural way of processing though it is not natural language processing.

The methodology of the process involved in the development of noosphereChardin (1948) where truths in the subconscious mind forms as part of our conscious mind as beliefs through experience when an individual spirit is formed which activates and controls a humanIssac (2010). The intersection of truths and beliefs is the knowledge created, which has an existence in the spirit, about which the Bible says about the Lord who forms the spirit in human (Zechariah 12:1). 
A CW based model, or simply CW model, has three principal components. (a) A question, Q, of the form: What is the value of a variable, Y? (b) An information set, I = $(\mathrm{p} 1, \ldots, \mathrm{pn})$, where the $\mathrm{pi}, \mathrm{i}=(1, \ldots, \mathrm{n})$, are propositions which individually or collectively are carriers of information about the value of $\mathrm{Y}$, that is, are question relevant. One or more of the pi may be drawn from world knowledge. A proposition, pi, plays the role of an assignment statement which assigns a value, vi, to a variable, $\mathrm{Xi}$, in pi. Equivalently, pi may be viewed as an answer to the question: What is the value of $\mathrm{Xi}$ ? $\mathrm{Xi}$ and vi, may be explicit or implicit. $\mathrm{Pi}$, is unconditional or conditional, expressed using if - then rule. An assignment statement constrains the values of $\mathrm{Xi}$. Here $\mathrm{Xi}$ is the constrained variable and vi is the constraining relation. More concretely, what this implies is that the meaning of a proposition, $\mathrm{p}$, may be represented as a generalized constraint, $\mathrm{X}$ is $\mathrm{R}$, in which $\mathrm{X}$ is the constrained variable, $\mathrm{R}$ is the constraining relation and $r$ defines the modality of the constraint, that is, the way in which $\mathrm{R}$ constrains $\mathrm{X}$. When vi is a word or combination of words, $\mathrm{Xi}$ is referred to as a linguistic variable, with vi being its linguistic value. When it is helpful to stress that pi assigns a value to a variable, pi is referred to as a valuation. Correspondently, the information set, I, is referred to as a valuation system, V. (c) The third component is an aggregation function, $\mathrm{f}$, which relates $\mathrm{Y}$ to the Xi. ie. $Y=f(X 1, \ldots, X n)$ Zadeh (2002) .

The principal difference between $\mathrm{CW}$ and conventional systems of computation is that $\mathrm{CW}$ allows inclusion in the information set, I, of propositions expressed in a natural language, that is, linguistic variations. Legalization of linguistic variations has important implications. The capability of computational methodologies to deal with imperfect information is greatly enhanced first, in which the information which in one or more respects is imprecise, uncertain, incomplete, unreliable, vague or partially true. When we look in to reasons behind this problem we can find that the factors described as the seven pillars of knowledge are the cause of imperfect computing. In realistic settings, such information is the norm rather than exception. Second, in cases in which there is a tolerance for imprecision, linguistic valuations serve to exploit the tolerance for imprecision through the use of words in place of numbers. And third, linguistic valuations are close to human reasoning and thus facilitate the design of systems which have a high level of machine intelligence, that is, high level of MIQ (Machine IQ) Zadeh (2002).Here we can find that wisdom is the factor which connects machines to natural intelligence. The wisdom factor is processed through the other six pillars of knowledge. Using the basic CW model, with the three principal components of the $\mathrm{CW}$ model, we can evaluate the values of Truth, Justice, Love, Loyalty, Obedience and Intelligence which must be delivered through another evaluation of the Wisdom factor, by simplifying in to two sets of three principal components.

Intelligence defined in many ways based on capacity for logic, abstraction, understanding, communication, learning, emotional knowledge, memory, creativity and problemsolving. It is described as the ability to perceive and retain knowledge or information that applies it to itself. It may also be the ability to perceive other instances of knowledge or information creating referable understanding models of any size, density, or complexity due to conscious or subconscious imposed will. In most simplified terms intelligence can be defined as what we do when we do not know what to do. Being intelligence is part of this geometric structure of knowledge, we can understand that intelligence have a key role in the generation of knowledge, which is generated through the spreading of words. Intelligence requires the support of data and information along with the basic logics on which a system is developed.

Knowledge representation (KR) incorporates findings from psychology and mathematics about how humans solve problems and represent knowledge in order to design formalisms that will make complex systems easier to design and build Maturana and Varela (1987).

Knowledge representation and reasoning incorporates findings from logic to automate various kinds of reasoning like the application of rules or the relations of sets and subsets Brachman(1978).Valuation-based system (VBS) is a framework for knowledge representation and inference. A network of interrelated entities, called variables are modeled in this framework by Prakash(1989).Knowledge level modeling is the process of theorizing over observations about a world and, to some extent,explaining the behavior of an agent as it interacts withits environment Menzies (1996).

Intelligence not only requires support of data andinformation, but it is using the knowledge itself tomove further based on logics. When we compareintelligence with other six pillars of knowledge,intelligence is more oriented towards Boolean logicsalong with the values of other pillars of knowledge.

Here our attempt to model knowledge brings out amodel for intelligence too as it is closely workingtogether with knowledge. When the knowledgespreads further, its direction is controlled throughintelligence and wisdom. As we already mentioned,intelligence is what we do when we do not know whatto do. The main focus of this paper is to bring out ageometric and physical modeling of naturalintelligence. Being the natural intelligence isassociated with the seven pillars of knowledge, themodel that we present here is giving a perfect modelfor knowledge and natural intelligence and thisknowledge exists in an eternal world Issac (2010).

\section{RESULTS AND DISCUSSION}

Intelligence defined in many ways based on capacity for logic, abstraction, understanding, communication, learning, emotional knowledge, memory, creativity and problemsolving. It can also be more generally described as the ability to perceive and/or retain knowledge or information and apply it to itself or other instances of knowledge or information. Johnston (2008).In most simplified terms intelligence can be defined aswhat we do when we do not know what to do. Beingintelligence is part of this geometric structure ofknowledge, we can understand that intelligence have akey role in the generation of knowledge, which isgenerated through the spreading of words. Intelligencerequires the support of data and information along withthe basic logics on 
which a system is developed.

This idea about the branch of science known asNoology is to be mentioned here, where the word isderived from the

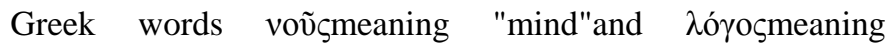
"logos".Noologyoutlines asystematic study and organization of everythingdealing with knowing and knowledge, i.e. cognitiveneuroscience. It is also used to describe the science ofintellect and intellectual phenomena. It is the study ofimages of thought, their emergence, their genealogy,and their creationIssac (2010). Logic, ideally the science ofreasoning, which is derived from the word Logos inGreek, is more concerned with the processes and products of reasoning. Moreover, the course of its development from Aristotle to the present day has been more idiosyncratic and specialized than what the concept of a science of reason would suggest.

In this paper we attempted to map the three components of $\mathrm{CW}$ in to a triangular form Issac (2013) and this triangular description is extended over a total of six triangles which is part of a hexagon word consisting of six sides along with seventh centroid and hence deductions are made with forming other hexagons which can be considered as the fundamental geometric and physical structure of a word which exists in an eternal world. As described in the Bible, like a honey comb, well ordered words are forming knowledge. This hexagon structure is the fundamental structure of pure water available in nature, hexagonal water, and like the structure of hexagonal snowflakes, which are formed like from a mould. Structurally and geometrically this hexagon formed out of six sub triangles with unifying centroid can be considered as the basic structure of a word. The three sides of each triangle can be considered as the propositions and the derivations drawn from the propositions through the process of CW Zadeh (2002).

This paper is also discussing about the physical and geometric structure of knowledge based on the seven pillars of knowledge Issac $(2012,2013)$ concept and how that is related with the natural intelligence and how we can model it to process with Artificial Intelligence to create more eco-friendly and user friendly machines world which can digest natural intelligence using CW (Computing with Words) of Cybernetics. The related field of artificial intelligence is the branch of computer science that endeavors to invest machines with mind and reason, or, ideally, that would create all possible types and degrees of intelligence. One of the natural sub fields of Noology should be modeling ideation, and of artificial intelligence the automation of ideation, but for some mysterious reason mere traces of these sub fields are all that can so far be found in those disciplines.

The main focus of this paper is to bring out a geometric and physical modeling of natural intelligence. Being the natural intelligence is associated with the seven pillars of knowledge, the model that we present here is giving a perfect model for knowledge and natural intelligence and this knowledge exists in an eternal world Issac (2010).

\section{CONCLUSION}

Cognitive psychologists use psychophysical and experimental approaches to understand, diagnose, and solve problems, concerning themselves with the mental processes which mediate between stimulus and response. They study various aspects of thinking, including the psychology of reasoning, and how people make decisions and choices, solve problems, as well as engage in creative discovery and imaginative thought. Cognitive theory contends that solutions to problems take the form of algorithms - rules that are not necessarily understood but promise a solution, or heuristicsrules that are understood but that do not always guarantee solutions Forester and Portage (2000). Cognitive science differs from cognitive psychology in that algorithms that are intended to simulate human behavior are implemented or implementable on a computer. Computing with Words (CW) is the latest research topic in Cybernetics related with this. In other instances, solutions may be found through insight, a sudden awareness of relationships. The ultimate is when we see visions and dreams at a sublimed level of thoughtIssac (2010). The approximations of these human businesses can be applied through the suggested natural intelligence modeling, once it is fully developed.

In coming years, computing with words and perceptions is likely to emerge as an important direction in science and technology. In a reversal of long standing attitudes, manipulation of perceptions and words which describe them is destined to gain in respectability. This is certain to happen because it is becoming increasingly clear that in dealing with realworld problems there is much to be gained by exploiting the tolerance for imprecision, uncertainty and partial truthZadeh (2002). This is the primary motivation for the methodology of computing with words (CW) Zadeh (2002). In this paper the author is trying to extract the potential of Computing with Words for processing with seven pillars of knowledge to give a geometric andphysical modeling of knowledge and hence naturalintelligence. We are publishing this paper incontinuation of the Government of India, UGCSponsoredMinor Research Project titled "Developmentof an indigenous Brain-Wave Machine", vide letter No.MRP(S)445/08-09/KLMG066/UGC-SWRO dated 30 ${ }^{\text {th }}$ March 2009

\section{REFERENCES}

[1] R. J. Sternberg and W. Salter, "Handbook of human intelligence. Cambridge", UK: Cambridge University Press. ISBN 0-521-29687-0.S. H. Rubin, "Computing with Words", IEEE Transactions On Systems, Man, And Cybernetics - Part B:Cybernetics, Vol.29, No.4, Pp.518524,1999

[2] L. A. Zadeh, "From Computing with Numbers to Computing with Words - From Manipulation of Measurements to Manipulation of Perceptions", International Journal of Applied Mathematics and Computer Science, Vol.12, No.3, Pp.307-324,2002.

[3] R. M. Issac, " Communication and Control through Words and Power", Advanced Materials Research, MEMS, NANO, and Smart Systems, Vol. 403, Pp.982-993,2012.

[4] R. M. Issac, " $\mathrm{CW}$ as a new computing technique in Electronic Applications", International Workshop on Recent Advances in Computing and Communications - 2013 (ACC-2013), 29-31 August 2013, Rajagiri School of Engineering \& Technology, Kochi, Kerala, India sponsored by IEEE, pp. 17-22, ISBN: 978-93-83083-25-1.

[5] The Bible

[6] P. T. Chardin, "The Phenomenon of Man", Harperperennial,1948

[7] J. Johnston, "The Allure of Machinic Life - Cybernetics, Artificial Lifeand the new AI", The MIT Press, 2008. 
[8] N. Wiener, "Cybernetics: or Control and Communication in the Animaland the Machine", The MIT Press, 1948.

[9] R. M. Issac, "Cybernetic perspectives of Desirable Practices in Teaching, Learning and Evaluation", Proceedings of the UGC Sponsored National Conference on Desirable Practices in Teaching, Learning \& Evaluation, at BPC College, Piravom, Kerala, Pp.46-77 2010.

[10] R. Brachman, "A Structural Paradigm for Representing Knowledge", Bolt, Beranek, and Neumann Technical Report ,1978.

[11] S. P. Prakash , "A valuation-based language for expert systems", International Journal of Approximate Reasoning, Vol. 3, No. 2, Pp. 383$411,1989$.

[12] T. Menzies. "Applications of Abduction: Knowledge-Level Modeling", 1996

[13] H. Maturana and F. Varela , "The Tree of Knowledge: The Biological Roots of Human Understanding”, Shambhala Publications, USA, 1987.

[14] A. D. Forester and M. R. Portage, "The Learners' Way: Brain-Based Learning in Action", 2nd edition, 2000, ISBN: 1894110552 